\title{
A Monte Carlo Study of Critical Properties of Strongly Diluted Magnetic Semiconductor (Ga,Mn)As
}

\author{
P. TOMCZAK ${ }^{a}$, H.T. DieP ${ }^{b, *}$, P. Jabeoński ${ }^{a}$ And H. Puszkarski ${ }^{a}$ \\ ${ }^{a}$ Faculty of Physics, Adam Mickiewicz University, Umultowska 85, 61-614 Poznań, Poland \\ ${ }^{b}$ Laboratoire de Physique Theorique et Modelisation, Universite de Cergy-Pontoise, CNRS, UMR 8089 2, \\ Avenue Adolphe Chauvin, 95302 Cergy-Pontoise Cedex, France \\ Within a Monte Carlo technique we examine critical properties of diluted bulk magnetic semiconductor \\ (Ga,Mn)As modeled by a strongly diluted ferromagnetic Heisenberg spin- $\frac{5}{2}$ system on a face centered cubic lat- \\ tice. We assumed that $5 \%$ of $\mathrm{Ga}$ atoms is substituted by $\mathrm{Mn}$ atoms and the interaction between them is of the \\ RKKY-type. The considered system is randomly quenched and a double average was performed: firstly, over the \\ Boltzmann probability distribution and secondly - over 2048 configurations related to the quenched disorder. We \\ estimated the critical temperature: $T_{c}=97 \pm 6 \mathrm{~K}$, which is in agreement with the experiment.
}

DOI: 10.12693/APhysPolA.133.514

PACS/topics: 75.50.Pp, 75.10.Nr, 75.30.Hx

Gallium manganese arsenide, (Ga,Mn)As, is a diluted magnetic semiconductor with a zinc-blende crystal structure with two interpenetrating FCC lattices (Ga and As). In one of them several percent of Ga is substituted, probably randomly, by Mn. This leads to the strong quenched site disorder. The compound is still focusing a lot of theoretical and experimental attention mainly due to its potential spintronic applications (i.e., a possible manipulation the spin and the charge carrier degrees of freedom at the same time). The effects of quenched disorder on critical properties of such systems have been the subject of intense experimental and theoretical interest for a long time. Generally, the critical temperature of bulk ( $\mathrm{Ga}, \mathrm{Mn})$ As depends on the concentration of $\mathrm{Mn}$ atoms, but details of this dependence are far from being known. Let us point to the recent [1] experimental answers, e.g., for the Mn concentration of $12 \%$ the value of $T_{\mathrm{C}}=183.5 \mathrm{~K}$ has been obtain for samples from remanent magnetization Kouvel-Fisher plots. On the other hand, using massive Monte Carlo simulations the critical properties of strongly disordered (but above the percolation threshold) Heisenberg systems (being model of (GaMn)As) on simple cubic lattice were examined [2]. Critical temperatures and critical exponents were estimated and it was shown, that Harris criterion is fulfilled.

In this paper we would like to elucidate the critical properties of strongly (the concentration of $\mathrm{Mn}$ atoms is below the percolation threshold) diluted (Ga,Mn)As. These systems are usually examined theoretically using a classical Heisenberg model,

$$
H=-\sum_{i, j} J\left(r_{i j}\right) \boldsymbol{S}_{i} \boldsymbol{S}_{j}
$$

$J\left(r_{i j}\right)$ stands for the hole-mediated, indirect exchange coupling between $\mathrm{Mn}$ moments separated by a distance $r_{i j}$ on FCC lattice,

$$
J(r)=J_{0} \mathrm{e}^{-r / l} r^{-4}(\sin (2 \kappa r)-2 \kappa r \cos (2 \kappa r)) .
$$

$\kappa$ is the Fermi wave number $\kappa=\left(\frac{3}{2} \pi^{2} n_{c}\right)^{1 / 3}, n_{c}$ stands for the hole density and $l$ is the damping scale.

Many authors have estimated exchange interactions entering to Eq. (2). Sato [3], using KKR method calculate $(\mathrm{Ga}, \mathrm{Mn})$ As electronic structure and subsequently embedding Mn impurities in CPA medium was able to to find the $J(r)$, see Fig. 1 (black squares). Fitting his data to the phenomenological formula given by Eq. (2) one can estimate the period of RKKY oscillations, $\sim \pi / \kappa \approx 1.37$ (cf. discussion in Ref. [4]).

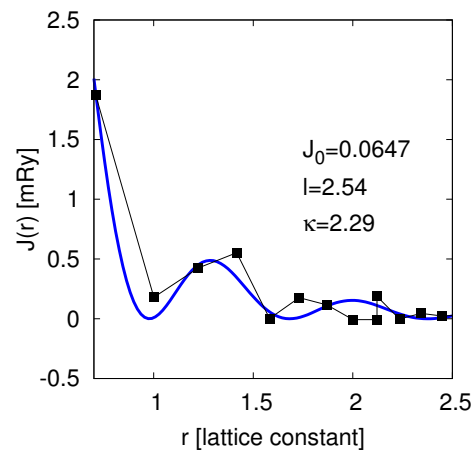

Fig. 1. Exchange interaction $J(r)$ in $(\mathrm{Ga}, \mathrm{Mn})$ As with $5 \%$ of Mn concentration versus distance, as calculated by Sato [3] (black squares). The blue line is a result of fitting Sato data to Eq. (2).

To obtain critical properties we have used an approach based on finite-size scaling hypothesis and analyzed the scaling of two quantities, which do not depend on the scale at which we look at the system: reduced correlation length $\frac{\xi}{L}$ and Binder cumulant $U_{2}$. As a test of this approach we have estimated the critical temperature and critical exponents $\nu$ and $\beta$ for classical FCC Heisenberg ferromagnet nearest neighbor interactions only. Firstly, 
let us recall the definition of the correlation length $\xi$ using the structure factors for two wave vectors: $\boldsymbol{q}=[0,0,0]$ and $\boldsymbol{q}_{\mathbf{1}}=\left[\frac{2 \pi}{L}, 0,0\right]$

$$
\xi=\frac{1}{\left|\boldsymbol{q}_{\mathbf{1}}\right|} \sqrt{\frac{S(\boldsymbol{q})}{S\left(\boldsymbol{q}_{\mathbf{1}}\right)}-1,}
$$

and the structure factor is given by

$$
S(\boldsymbol{q})=\sum_{\boldsymbol{r}} \cos (\boldsymbol{q} \cdot \boldsymbol{r}) C(\boldsymbol{r}),
$$

with $C(\boldsymbol{r})$ being the correction function at the distance $|\boldsymbol{r}|$. Subsequently, using the Metropolis algorithm (200000 MC steps/spin, 10000 steps/spin to reach equilibrium) we have calculated $\xi(T)$ for three FCC systems: $L=10,12,14$ consisting of 4000,6912 and 10976 spins, respectively, with periodic boundary conditions.
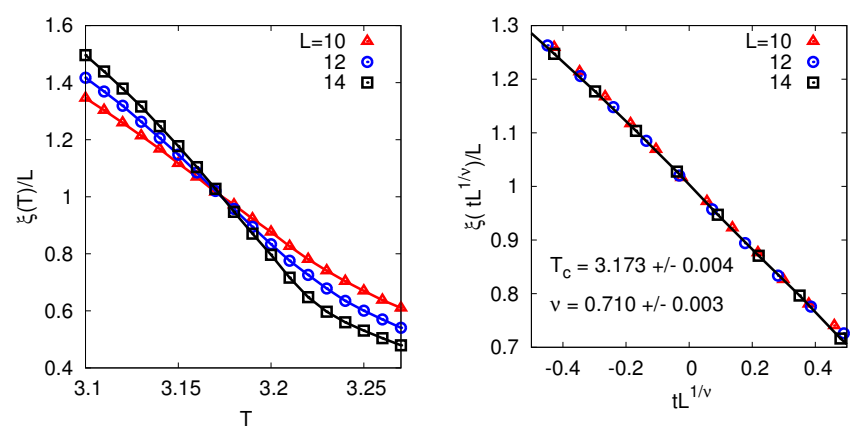

Fig. 2. Temperature dependence of the three correlation lengths normalized by the system size $L$ in three classical Heisenberg FCC systems before rescaling (left) and after rescaling (right). $t$ stands for the reduced temperature, $\frac{T_{c}-T}{T}$. The scaling collapse leads to the optimal values of $T_{c}$ and exponent $\nu$. Statistical errors are comparable with points sizes.

Since one assumes that a quantity which is singular at $T_{c}$ in the thermodynamic limit, scales with the system size $L$ close to $T_{c}$ as a power of $L$ multiplied by a nonsingular function of the ratio $\frac{\xi}{L}$, one has

$$
\xi=L g\left(t L^{\frac{1}{\nu}}\right)
$$

where $g$ stands for a scaling function. Thus, an attempt to plot $\xi$ vs $t L^{\frac{1}{\nu}}$ with a proper values of $T_{c}$ and $\nu$ should result in a scaling collapse - this is shown in Fig. 2 (right).

Besides examining the scaling of $\frac{\xi}{L}$ there exists other dimensionless size-independent quantity at the critical point which can be used to extract critical properties of the system under consideration, namely the Binder $U_{2}$ cumulant. In the case of classical Heisenberg system (or a vector order parameter) it is defined in the following way:

$$
U_{2}=\frac{5}{2}\left(1-\frac{3}{5} \frac{\left\langle m^{2}\right\rangle}{\langle|m|\rangle^{2}}\right) .
$$

During the same MC run we have calculated $\left\langle m^{2}\right\rangle$ and $\langle|m|\rangle$. The scaling of $U_{2}$ cumulant enables to extract a critical exponent $\nu$ for the second time from the scaling of the function $h$

$$
U_{2}=h\left(t L^{\frac{1}{\nu}}\right) .
$$

This is shown on the left side of Fig. 3 - before rescaling and on the right side - after rescaling.
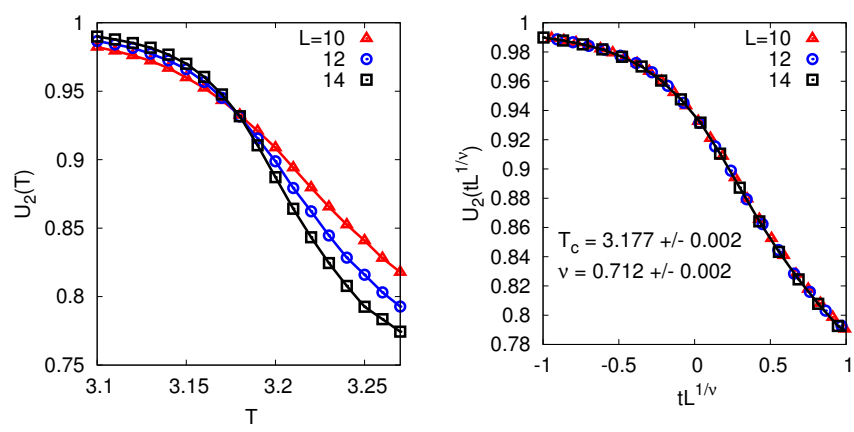

Fig. 3. The cumulant $U_{2}$ before (left) and after rescaling (right). As expected from the finite size scaling ansatz (Eq. (7)) the data for different system sizes collapse on a single curve for $T_{c}=3.177 \pm 0.002$ and $\nu=0.712 \pm 0.002$ (right).

It is also possible to estimate the second independent exponent $\beta$ from the following scaling of the magnetization

$$
m(t, L)=L^{-\frac{\beta}{\nu}} f\left(t L^{\frac{1}{\nu}}\right),
$$

with $f$ being some scaling function. Plotting $m(t, L) L^{\frac{\beta}{\nu}}$ versus $t L^{\frac{1}{\nu}}$ for different system sizes leads to the scaling collapse for $\frac{\beta}{\nu}=0.518 \pm 0.001$, see Fig. 4. Our numerical values should be compared $[5,6]$ with $T_{c}=3.1771 \pm$ $0.0001, \nu=0.7112 \pm 0.0005$ and $\beta=0.3689 \pm 0.0003$.
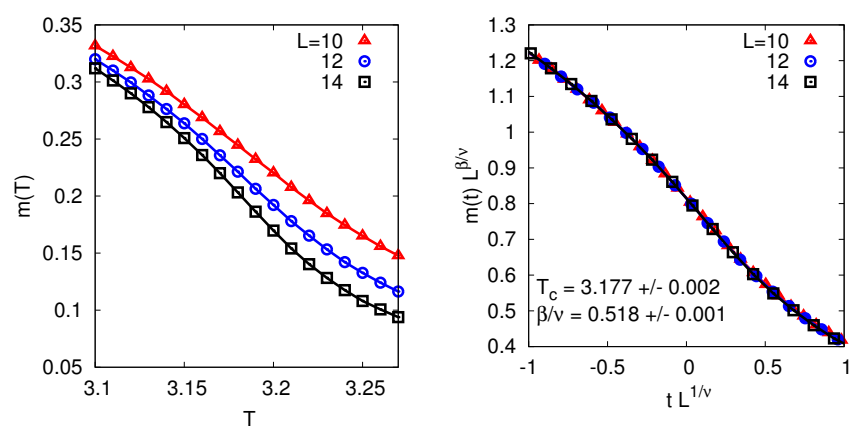

Fig. 4. The magnetization $m(T)$ before (left) and after rescaling (right). As expected from the finite size scaling ansatz (Eq. (8)), the data for different system sizes fall onto a single curve for $T_{c}=3.177 \pm 0.002$ and $\frac{\beta}{\nu}=$ $0.518 \pm 0.001$ (right).

Let us now move to a more complicated structure, namely to a strongly diluted classical Heisenberg FCC system with long-range $R K K Y$-type interactions, given by Eq. (2). Suppose that a concentration of magnetic $\mathrm{Mn}$ atoms is equal to 0.05 which is far below the percolation threshold $p_{0}$ for the nearest neighbor Heisenberg system $\left(p_{0}=0.20\right.$ for the FCC lattice).

The system is a randomly quenched one and therefore one has to take a double average: for fixed configuration 
of magnetic atoms, see Fig. 5, one computes the thermal average within the Metropolis importance sampling and subsequently, the average over disorder is realized by simple sampling.

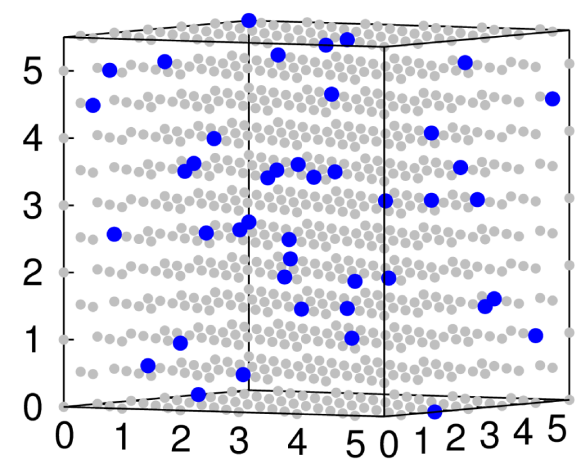

Fig. 5. One of the possible configurations of magnetic atoms chosen from $6^{3} \cdot 4$ atoms (grey) in FCC structure. Only $5 \%$ of them are magnetic ones (blue). Averaging over such configurations of magnetic atoms one should take into account $\sim 10^{4}$ of them.

One could ask a basic question how large should be the sample which will be used for averaging over configurations. Samples being examined in typical experiments amount a very large number of degrees of freedom $\left(\sim 10^{23}\right)$ and the observable quantities are selfaveraging [7]. the situation is quite different in the case of a finite size systems and finite size scaling analysis. The main problem is that by considering systems of finite linear dimension $L$ at the critical temperature $T_{c}$ one encounters fluctuations by passing from one configuration to another which cause a significant fluctuation of the pseudo-critical temperature $T_{c}$. This implies that one has to average over $\sim 10^{4}$ configurations in order to get the relative error of the disorder average at $T_{c}$ less than $1 \%[7]$.
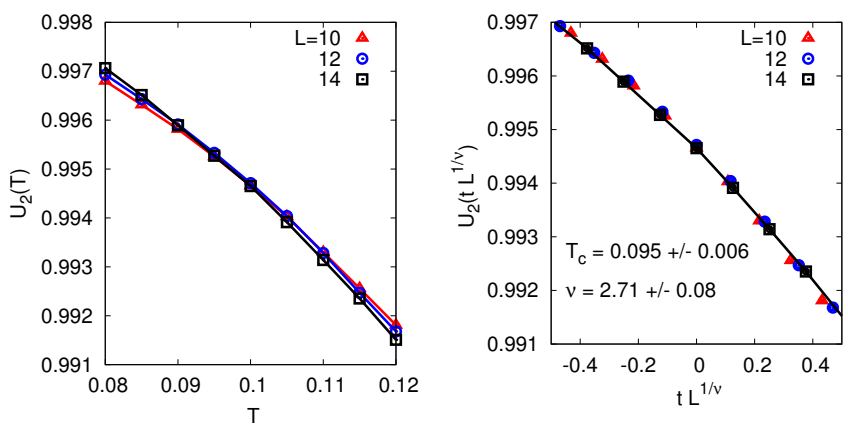

Fig. 6. The magnetization $m(T)$ for diluted systems before (left) and after rescaling (right). The data for different system sizes fall onto a single curve for $T_{c}=$ $0.095 \pm 0.006$ and $\frac{\beta}{\nu}=0.370 \pm 0.050$ (right). The concentration of Mn atoms amounts $5 \%$, interactions [3] are given in Fig. 1, black squares.
Let us summarize the examination of the critical properties of (Ga,Mn)As with 5\% Mn atoms and interactions between them presented in Fig. 1. The average values of $m(T)$ and $U_{2}(T)$ were calculated for 2048 configurations. It was not possible to find a proper scaling dependencies $m(T)$ - statistical errors resulting from fluctuations were much bigger than differences between $m_{10}(T), m_{12}(T)$ and $m_{14}(T)$. However, it was possible to find a proper scaling for correlation length $U_{2}(T)$. The effect (crossing in Fig. 6 (left)) is rather subtle but clearly marked and it is definitely beyond the statistical error. Taking into account that $1 \mathrm{Ry}=158 \mathrm{~K}$ and $S=\frac{5}{2}$ for $\mathrm{Mn}$, one can estimate $T_{c}$ for (Ga,Mn)As containing of $5 \% \mathrm{Mn}$ atoms as follows: $T_{c}=158 \cdot\left(\frac{5}{2}\right)^{2} \cdot 0.095=97 \pm 6 \mathrm{~K}$ which is in agreement with experiment [1]. Note that both types of data, presented in Fig. 1 lead to the same (within a statistical error) value of $T_{c}$. A high a value of the exponent $\nu=2.71$ may indicate that the considered transition belongs to a universality class different from that of the 3D Heisenberg. Possible long-range RKKY interactions and strong site quenched dilution might be the cause of this difference. Similar behavior was observed [8] in case Heisenberg spin glasses. We leave this issue open for future investigation.

\section{Acknowledgments}

This work was a part of a project financed by Narodowe Centrum Nauki (National Science Centre of Poland), Grant No. DEC-2013/08/M/ST3/00967. Numerical calculations were performed at Poznań Supercomputing and Networking Center under Grant No. 284.

\section{References}

[1] M. Wang et al., Phys. Rev. B 93, 184417 (2016).

[2] D.J. Priour, S. Das Sarma, Phys. Rev. B 81, 224403 (2010).

[3] K. Sato et al., Phys. Rev. B 70, 201202R (2004).

[4] T. Dietl, H. Ohno, Rev. Mod. Phys. 86, 187 (2014).

[5] A. Pelissetto, E. Vicari, Phys. Rep. 368, 549 (2002).

[6] J. Adler, Ch. Holm, W. Janke, Physica A 201, 581 (1993).

[7] D.P. Landau, K. Binder A Guide to Monte Carlo Simulations in Statistical Physics, Cambridge University Press 2014.

[8] H. Kawamura, J. Phys: Conf. Series 233, 012012 (2010). 